\title{
The Report of 20 Missed Real COVID-19 Cases
}

ISSN: 2639-0531

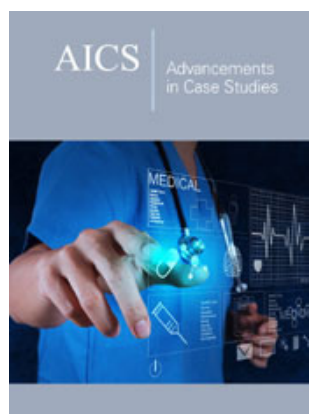

*Corresponding author: Iraj Salehi Abari, Rheumatology Research Center, Tehran University of Medical Sciences, Iran

Submission: 洞 August 21, 2020

Published: 此 September 28, 2020

Volume 2 - Issue 5

How to cite this article: Iraj Salehi Abari, Shabnam Khazaeli. The Report of 20 Missed Real CovID-19 Cases. Adv Case Stud. 2(5). AICS.000546. 2020. DOI: 10.31031/AICS.2020.02.000546

Copyright@ Iraj Salehi Abari, This article is distributed under the terms of the Creative Commons Attribution 4.0 International License, which permits unrestricted use and redistribution provided that the original author and source are credited.

\author{
Iraj Salehi Abari ${ }^{1 *}$ and Shabnam Khazaeli ${ }^{2}$ \\ ${ }^{1}$ Rheumatology Research Center, Tehran University of Medical Sciences, Iran \\ ${ }^{2}$ General Practitioner, Genetic Consultant, Canada
}

\begin{abstract}
The COVID-19 is a new contagious viral/immunological systemic disorder with predominantly respiratory features caused by human infection with SARS-CoV-2. From the time the virus enters the body to the time of initial symptoms of the disease, it is called the incubation period which ranges from one day to fourteen days. Initial presentation in the patients with mild COVID-19 is including mild fever, dry cough, fatigue and then body ache, anosmia, and lack of taste. About $40 \%$ of patients with COVID- 19 are asymptomatic and, 40\%; mild, 15\%; severe, and 5\% are critical COVID-19. A negative SARS-CoV-2 test does not rule out the diagnosis of COVID-19 and that a positive test does not necessarily indicate a COVID-19 diagnosis. The normal lungs in HRCT scan with a negative SARS-CoV-2 test does not preclude that the person has COVID-19. Patients may contract COVID-19 without any risk factors and have no symptoms. The authors conclude that to reject or confirm the diagnosis of COVID-19 by performing a SARS-CoV-2 kit test and the HRCT scanning of lungs may not be the gold standard for diagnosis of COVID-19. In other words, history is the key to COVID-19 diagnosis. Therefore, in this article, twenty cases of COVID-19 are presented, in most of which the diagnostic test of SARS-CoV-2 and HRCT scan of the lungs are negative.
\end{abstract}

Keywords: SARS-CoV-2 test; COVID-19; HRCT scan of lungs

\section{Introduction}

The novel Coronavirus disease or COVID-19 is a new contagious viral/immunological systemic disorder with predominantly respiratory features caused by human infection with SARS-CoV-2, which is rapidly spreading from person-to-person all around the world as a pandemic. This virus is super-saturated for hours to days in many places/spaces, so that a moment's presence and breathing in such places/spaces is enough to get COVID-19. Not only sneezing, coughing, shaking hands and kissing can cause COVID-19, but also talking and breathing normally and even gas passing from the intestine can cause the disease [1-3]. From the time the virus enters the body to the time of initial symptoms of the disease, it is called the incubation period which ranges from one day to fourteen days. Initial presentation in the patients with mild COVID-19 is including mild fever, dry cough, fatigue and then body ache, anosmia, and lack of taste. About $40 \%$ of patients with COVID-19 are asymptomatic and, $40 \%$; mild, 15\%; severe, and 5\% are critical COVID-19 [4-6]. Real-Time, Reverse Transcriptase Polymerase Chain Reaction test, or in short RT-PCR test is the gold-standard diagnostic test for the detection of COVID-19. A positive RT-PCR test in a patient with viral symptoms/signs in ENT and lung with or without other organ's involvement indicates the presence of an active COVID-19. However, a positive RT-PCR in an asymptomatic person suggests three conditions including subclinical COVID-19, pre-symptomatic COVID-19 within the incubation period, and carrier state. It can also show false-negative results in; late phase of inactive COVID-19, very early phase of COVID-19 in which the virus replication has not yet started, a case of COVID-19 in which the sample is taken from a site that does not contain the virus or sample is collected with errors [4-6]. A positive COVID-19 IgM antibody test shows both active COVID-19 and recently improved COVID-19, whereas a positive COVID-19 IgG antibody test 
not only shows the recent previous COVID-19 but also reveals the body's immune protection against re-infection [4-6]. Elevated ESR/ CRP along with low lymphocyte count and abnormal liver function tests are compatible with mild to moderate cases of COVID-19 [4-6]. All symptomatic individuals who are suspected of having COVID-19, should undergo Chest-X-Ray, and even if CXR is normal; in them HRCT scanning of lungs is recommended. All asymptomatic individuals who have a positive result for COVID-19 diagnostic test should undergo imaging too. It is important to know that during the first four days of the illness, lung involvement in imaging is limited to one lobe in one-third of cases and not seen at all in one-fifth of cases [4-6]. We need to know that getting a very accurate history is the mainstay in the diagnosis of COVID-19, while COVID-19 diagnostic tests and HRCT scanning of lungs are tools to confirm or reduce it [4-6].

A positive history including acute respiratory symptoms following a recent trip to a red zone in novel Corona epidemic or recent contact with a patient of COVID-19 strongly is in favor of the diagnosis of COVID-19 even if the COVID-19 diagnostic test is negative and lung imaging is normal. Knowledge of the list of clinical findings and risk factors for COVID-19 is essential for screening and diagnosis. The list of Clinical findings includes: fever, dry cough, hoarseness, shortness of breath, fatigue, sneezing, runny nose, sore throat, nasal congestion or stuffy nose, sputum, chest pain, anosmia and lack of taste, cold or flu diagnosis over the past four weeks. The list of Risk factors includes: contact with a COVID-19 patient within recent four weeks, travelling to Red zones within the recent four weeks, working in the live animal market, contact with a patient who has died due to unknown pneumonia within recent 4 weeks, contact with a live animal market worker, and failure to follow the principles of prevention of becoming infected with SARS-CoV-2, contact with someone who has traveled to Red zones within recent four week, living in Red zone of the city, contact with someone who has contacted with a COVID-19 patient within recent four weeks, and maybe close contact with pets [4-6].

\section{Introducing 20 real cases of COVID-19: Do you think the following patients have COVID-19?}

A. Case (1): A 66-year-old woman without any past history due to high fever and confusion, has been hospitalized. After HRCT scanning, both lungs are bleached, but RT-PCR is negative.

B. Case (2): Anold man keptin a nursing home. Hehas diabetes mellitus. Without previous history, he suffers from lethargy and respiratory distress. In portable radiography, multiple opacities are evident in both lungs, so he is hospitalized, in blood tests, ESR and CRP are very high, along with low lymphocyte count, he died within 24 hours, despite being connected to a ventilator machine and prescribed broad-spectrum antibiotics. RT-PCR is also negative.

C. Case (3): A 45-year-old woman who was nursing her mother, who suffers from COVID-19, developed a dry cough with normal physical examination a week later. In Chest radiography there are multiple consolidations, but RT-PCR is negative.
D. Case (4): A 70-year-old man who visited a place of pilgrimage ten days ago has lost his sense of smell and taste. Due to chest pain and following a plain CXR and taking an ECG and a SARS-CoV-2 diagnostic test, only bilateral hazy lungs is evident.

E. Case (5): Occurrence of severe dry cough since yesterday with normal lungs examination in a physician is a member of the team of physicians at Corona Hospital for COVID-19. His RTPCR is negative.

F. Case (6): A young tourist with loss of taste and lack of smell after traveling to Wuhan within 2 recent weeks with the normal evaluations.

G. Case (7): After returning from a trip to Iran, despite any complaints and the normality of clinical examinations on the recommendation of the doctor, she is checked up that only the HRCT scan of lungs shows a turbidity limited to a lobe in the lungs.

H. Case (8): A 30-year-old women, after staying at home for 4 months, finally overcomes her emotions with reason and decides to attend a prayer ceremony in the neighborhood mosque. Twelve days after this ceremony, she does not wake up in bed in the morning, which confirms her death with the advice of the family physician, while she was completely healthy until the night before. Tests of samples taken from the deceased indicated that the COVID-19 IgG test was positive, but both the COVID-19 IgM and RT-PCR tests were negative.

I. Case (9): One week after an Iranian businessman meets with a businessman who returned from China 10 days ago, he is checked up at his family doctor's insistence, despite no clinical findings. All tests were negative except for leukopenia and very high CRP, which were not present in previous experiments.

J. Case (10): A young man has a severe cough after returning from a pilgrimage. Despite the normality of the examinations, everything is reported to be normal in his evaluations, and the only positive finding is the COVID-19 IgM test, despite the negative RT-PCR.

K. Case (11): An university entrance exam candidate after check-up tests one week before taking the national entrance exam finds that he has a very high CRP and the RT-PCR test is positive. That is why he and another group of volunteers will be tested in a separate place.

L. Case (12): A young pedestrian was brought to the emergency room and then ICU, following the coma caused by a landslide and a skull hit on the pavement. Except for the coma, other physical examinations are normal, and after identifying the person and informing and referring one of the patient's first-degree relatives, it is determined that there was no problem in his history. To be examined for traumatic brain injury, he undergoes a brain MRI, which is reported to be normal. Chest radiograph shows hazy lungs that during HRCT 
scan of the lungs, white lungs is seen, and then the RT-PCR test is positive.

M. Case (13): A chef immediately sees an ENT specialist to check and fix any problems due to loss of sense of taste and smell for fear of losing his job. There are no positive findings in the history and physical examination. The only positive finding in the evaluations was the observation of multiple consolidations in the CT scan of lungs.

N. Case (14): Following the death of a family member due to pneumonia and a positive SARS-CoV-2 test report after death, other family members undergo clinical and para-clinical evaluations. Only on HRCT scan of the lungs in the youngest member of the family, a 12-year-old boy, the image of white lungs is seen without any other positive findings.

0. Case (15): A 40-year-old woman sees an Ophthalmologist because of redness in both eyes without itching, pain, and blurred vision. The clinical finding is acute bilateral follicular conjunctivitis. Due to the SARS-CoV-2 epidemic, the Ophthalmologist consults the patient with an Infectious Diseases Specialist for COVID-19 evaluations. The diagnosis of COVID-19 is made only by observing white lungs on an HRCT scan of the lungs. Case (16): Following routine reviews in the administrative recruitment test; positive COVID-19 IgG test, negative IgM, negative RT-PCR, and white lungs on chest radiography prevented recruitment due to COVID-19.

P. Case (17): A 30-year-old man goes to a nearby clinic, because of mild abdominal pain and diarrhea. Initial clinical and para-clinical evaluations were normal. No medication is prescribed, while connecting one liter of saline/sugar solution and healing the patient. The next day, due to the recurrence of the above symptoms, he went to an Infectious Diseases Specialist, that by conducting tests; RT-PCR is negative, but COVID-19 IgM test is positive.

Q. Case (18): The family grandfather dies of fever and coma. Despite the negative RT-PCR of the deceased, all family members are also screened, which the COVID-19 test is reported to be positive in the father along with high CRP and low leukocyte count. His RT-PCR was negative.

R. Case (19): A 20-year-old girl is examined one week after having sex with her boyfriend on the advice of a physician. In addition to a negative pregnancy test, COVID-19 IgM test is positive and high CRP and low leukocyte count are reported, but the reported RT-PCR is negative.

S. Case (20): After attending the funeral of the family's older brother in Tehran, a significant number became infected with COVID-19, and six of them died in less than a week. One of the participating members dies a week later, despite being a 21-year-old healthy man and having a negative RT-PCR test, but high CRP and leukopenia.

\section{Discussion}

Like other diseases in internal medicine, the clinical/paraclinical judgment of an expert Internist and/or Infectious Disease Specialist is the gold standard for diagnosis of COVID-19, of course in consultation with other medical professionals. We all know that a negative SARS-CoV-2 test does not rule out the diagnosis of COVID-19 and that a positive test does not necessarily indicate a COVID-19 diagnosis, and it is positive in carriers of virus too. Also, the normal lungs in HRCT scan, even with a negative SARS-CoV-2 test cannot rule out COVID-19. Many patients have COVID-19 without any risk factors. And many patients have no symptoms but have COVID-19. Therefore, it is simplistic for us to reject or confirm the diagnosis of COVID-19, only by performing a SARS-CoV-2 kit test and the HRCT scanning of lungs. For these reasons, the corresponding author concluded that to regulate the diagnosis of COVID-19 and to establish the same and coordinated decisions around the world regarding the diagnosis of COVID-19, it is necessary to provide a very sensitive and accurate Diagnostic Criteria for diagnosing this disease.

\section{Conclusion}

Negative medical history, normal clinical examination, negative SARS-CoV-2 kit tests, and normal HRCT scanning of the lungs alone and even all together cannot rule out the diagnosis of COVID-19. But of all the above tools, medical history is the most reliable way to identify COVID-19 and its diagnostic key. Based on the above facts, the corresponding author of the article (ISA) has provided a Diagnostic Criteria for COVID-19, which is called the Persian Gulf Criteria [4].

\section{References}

1. Gu JY, Han B, Wang J (2020) COVID-19: Gastrointestinal manifestations and potential fecal-oral transmission. Gastroenterology 158(6): 15181519.

2. Cicuttin E, Cobianchi L, Chiarugi M, Catena F, Coccolini F, et al. (2020) Detect to protect: Pneumoperitoneum gas samples for SARS CoV2 and biohazard testing. Surgical Endoscopy 34: 2863-2865.

3. World Health Organization (2020) Transmission of SARS-CoV-2: Implications for Infection Prevention Precautions: Scientific Brief. WHO.

4. Salehi-Abari I, Khazaeli S, Salehi-Abari F, Salehi-Abari A (2020) Practical guideline for screening the patients with SARS-CoV-2 infection and Persian Gulf criteria for diagnosis of Covid-19. Advances in Infectious Diseases 10: 67-93.

5. Coronavirus: Information \& resources. National Jewish Health Coronavirus.

6. WHO (2020) News and events regarding COVID-19 and SARS CoV-2.

For possible submissions Click below: 